\title{
Parameter Estimation of Drag Coefficient and Rolling Resistance of Vehicles Based on GPS Speed Data
}

\author{
A. SzÁNTÓ1 ${ }^{1}$ S. HAJDU ${ }^{2}$ \\ ${ }_{1}^{1}$ University of Debrecen, Faculty of Engineering, andras.szanto.0503@gmail.com \\ 2University of Debrecen, Faculty of Engineering, hajdusandor@eng.unideb.hu
}

In this paper, a parameter estimation method of the model-based design approach is applied to estimate the drag coefficient and the rolling resistance coefficient of a vehicle. In fact, a constant-force parameter (c_const) and a velocity-square-force parameter (c_square) are in the vehicle model, and these result in the sum force applied along the translational DOF that models the vehicle. It is only an assumption that the constant force is the rolling resistance and the force proportional to the square of the velocity is the drag force of the air. Only GPS speed data is used for the estimation process. The conclusion is that parameter estimation is a good alternative when expensive measurement devices are not available to measure the force losses separately and directly.

\section{Introduction}

Parameter estimation [1,2] is a key method in model-based design (MBD) [3,4]. In MBD, we need validated mathematical models of the parts of a system. The mathematical models are usually lumpedelement models [5] instead of distributed-parameter models [6]. Parameter estimation is needed when the constant parameters of the lumped-elements cannot be computed directly from the measurement data. This happens when the accessibility of measurement devices is limited. In this case, parameter estimation is applied which is an optimization process: a cost function is calculated based on the difference of the measurement data and the same data in the mathematical model, and the variables of the optimization are the appropriate constant parameters of the elements in the model. The estimation is successful if the fit of the curves is acceptable. Then, the estimated parameters need to be validated with different measurements. The main environments for lumpedelement modelling and parameter estimation (and a lot of other things and methods) are MATLAB, Simulink and Simscape, all of them developed by MathWorks.

In [7] detailed description of drag can be found. Although a force proportional to the square of the velocity is a common way to model air resistance, in the low Reynolds number region a linear model is more appropriate (Stoke's drag). In [8] road grade and vehicle parameters of heavy vehicles are estimated based on GPS and other data.

The authors previously researched lumped-element vehicle models in Simscape based on the Pacejka semi-empirical model [9], and its application for modelling a theoretical ABS algorithm [10]. 


\section{Vehicle model in details}

The vehicle model has one degree of freedom: the longitudinal translational freedom. An additional rotational freedom is needed if the driven wheels are intended to be modelled. In the case of this project, there are not enough data to estimate the parameters of a wheel model, therefore it is not modelled. Although the rolling resistance torque is in connection with the wheels, in this model it is considered as a force which directly affects the translational freedom of the body.

Not only the rolling resistance torque but other wheel forces and torques are modelled by the Pacejka wheel models [9]. The authors previously researched lumped-element vehicle models in Simscape based on the Pacejka semi-empirical model, and its application for modelling a theoretical ABS algorithm [10]. This previous vehicle model was modelled with several DOF: not only had sophisticated wheel models but the suspension of the vehicle and road unevenness were also considered. Mentioning this reference, I would like to point out that the vehicle model for this estimation is purposely simple. The reason for this simplification is that the measurement devices and sensors are very limited (for this project) and there is no use in using complex models if their parameters cannot be estimated.

Differential equation of the vehicle model:

$$
\mathrm{m} * \mathrm{a}=\frac{1}{2} \mathrm{v} *|\mathrm{v}| * \mathrm{c}_{\text {square }}+\mathrm{m} * \mathrm{c}_{\text {const }}
$$

where $a$ is the acceleration, $v$ is the velocity, $m$ is the mass, $c_{-}$const is the constant force parameter and $c_{-}$square is the velocity-square force parameter.

It is only an assumption that the constant force is the rolling resistance and the force proportional to the square of the velocity is the drag force of the air.

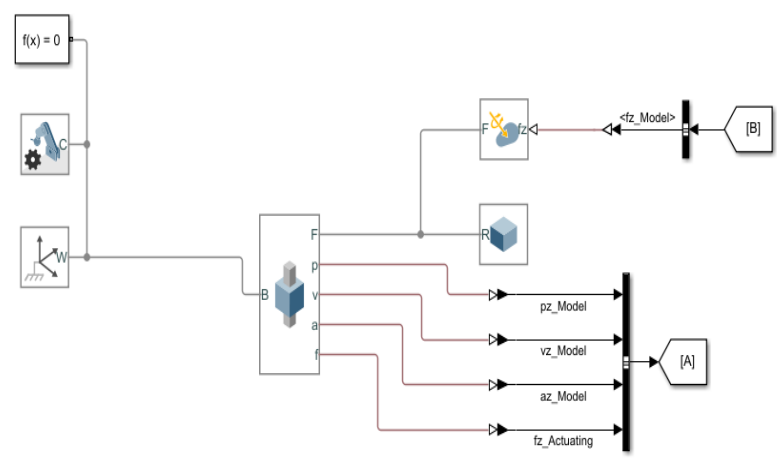

Figure 1: Vehicle model in Simscape Multibody

\section{Measurement in details}

The only device was used for data acquisition is a phone. Although a phone has several sensors apart from GPS, for the purpose of this estimation only the GPS speed data was used. The reason for this is 
that the inertial measurement units (IMU sensors) cannot measure velocity directly. Sensor fusion methods could be used to get a more precise velocity data than the pure GPS speed data, but in this paper the method to get the necessary velocity data was not in the focus. The GPS speed data is available with $1 \mathrm{~Hz}$ frequency. The estimated vehicle is a bicycle, $\mathrm{m}=120 \mathrm{~kg}$.

In the figure below, one complete measurement can be seen. It has 3 phases:

- The measurement starts when the vehicle has zero velocity.

- In the acceleration phase, the vehicle is accelerated to a specific top speed.

- In the deceleration phase, the vehicle is decelerated by only the force losses (energy losses). Based on the vehicle model and the data of the deceleration phase, the constant parameters of the forces can be estimated.

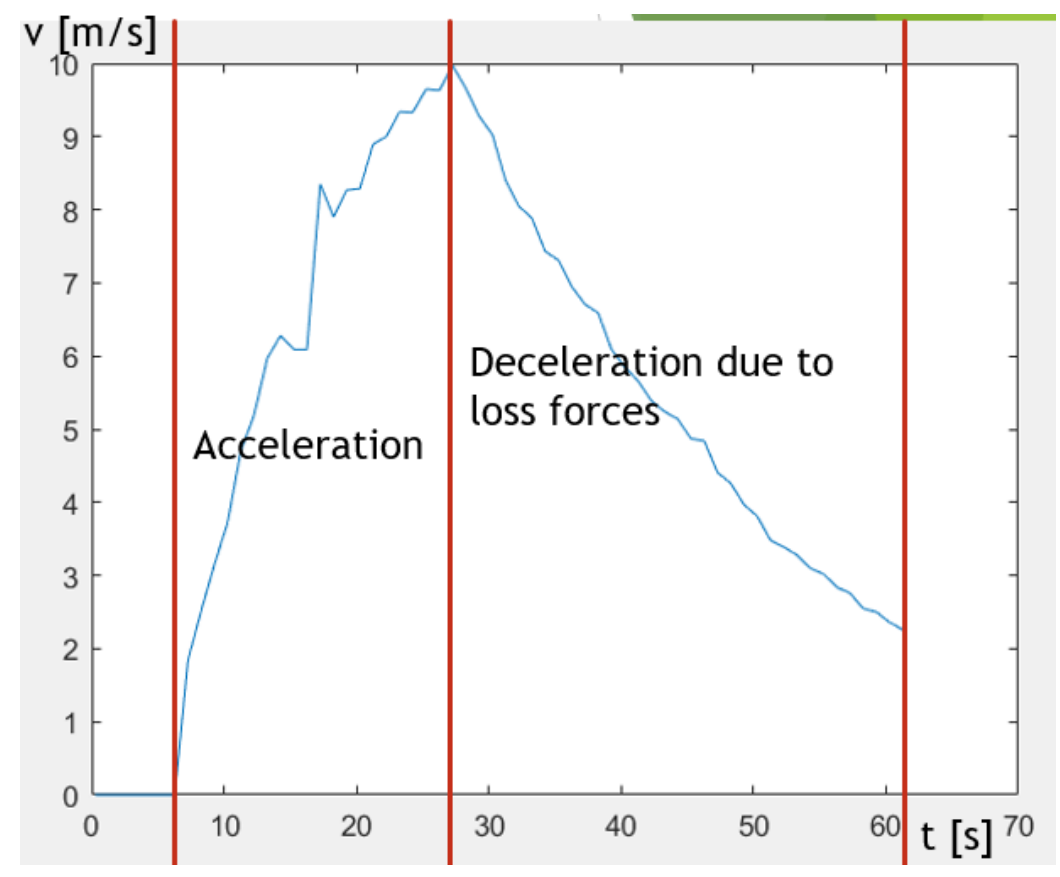

Figure 2: One complete measurement

There were 3 different conditions for the measurement:

- $\quad 5$ bar \& straight: 5 bar tire pressure and "normal position" of the driver's body

- 5 bar \& down: 5 bar tire pressure and "aerodynamic position" of the driver's body

- 1 bar \& straight: 1 bar tire pressure and "normal position" of the driver's body

\section{Estimation and results}

\section{Essence of parameter estimation:}

- Create objective function that is to be minimized: from now it is an optimization problem.

- The objective is based on the difference of the measured data and the simulated data. 
International Journal of Engineering and Management Sciences (IJEMS) Vol. 5. (2020). No. 2

DOI: 10.21791/IJEMS.2020.2.14.

- Usually this difference is squared ("least squares") point by point then summed.

- Define the variables of the optimization.

- The variables are the constant parameters that are to be estimated.

- Solve the optimization with optimization algorithms.

Application used for this problem: Simulink Parameter Estimation application. It is based on MATLAB optimization functions, with a simple GUI. For custom optimizations/estimations, one can run Simulink models from MATLAB code.

The results are presented in the following table.

\begin{tabular}{|l|l|l|l|}
\hline variables/ conditions & 5bar \& straight & 5bar \& down & 1bar \& straight \\
\hline c_const Avg & -0.04526 & -0.05647 & -0.1444 \\
\hline c_square Avg & -0.8155 & -0.4496 & -0.6961 \\
\hline
\end{tabular}

Figure 3: Results of the estimations

\section{Evaluation of the results:}

- In theory:

- The driver's body position affects only the $c_{-}$square parameter.

- The tire pressure affects only the $c_{-}$const parameter.

- The results show good agreement with the theory, but not perfect.

- Possible reasons: models are not perfect (the rolling resistance is not perfectly constant / linear Stokes-drag below a speed limit [7]), or there were not enough samples (only 2 per condition).

Checking the optimization output: it is necessary to check that if the given local minimum is valid.

- The $\mathrm{f}(\text { c_const,c_square })_{-}$objective function is evaluated in the output point and in its environment of $1 \mathrm{e}-2$.

- Objective function: $f(x)=\sum_{i} e_{i}(x)^{2}$, where $e$ is the error in one measurement point. 

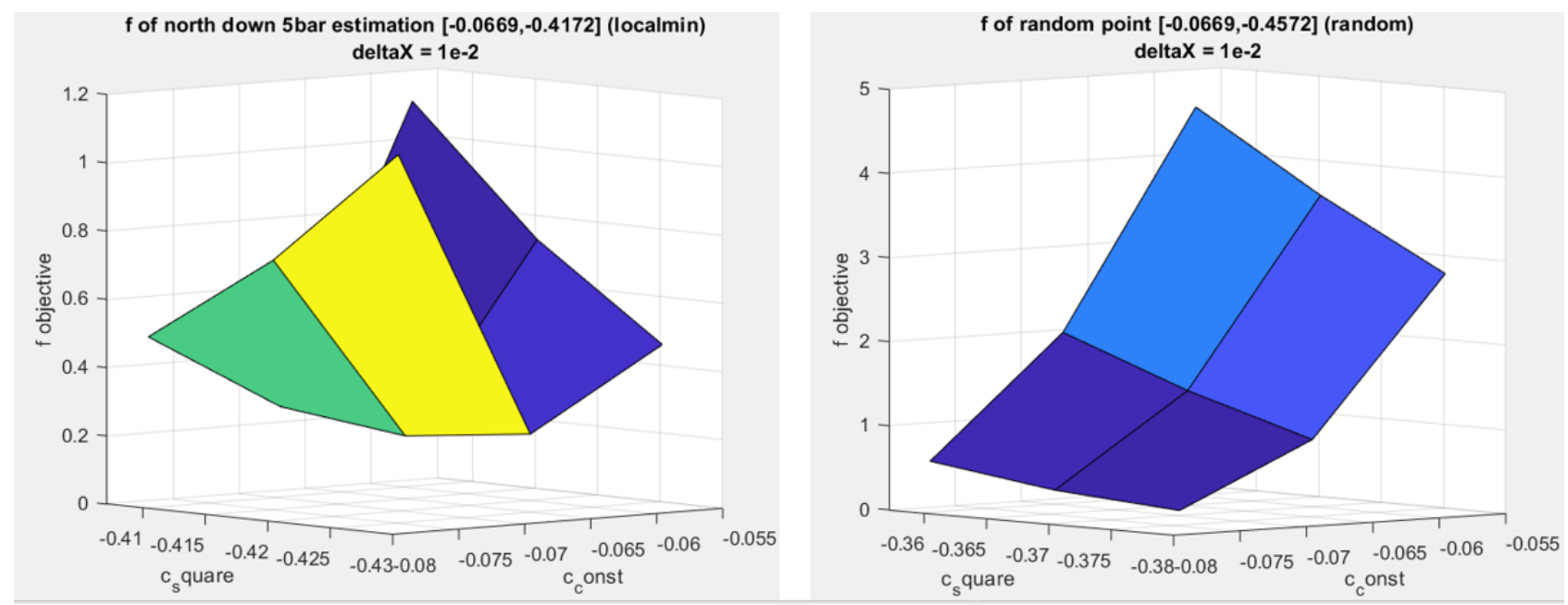

Figure 4: Objective function evaluation in the optimization output point (left) and a random point (rigth) and in their environments. It can be seen that the optimization output point is a valid local minimum.

\section{Experiment: estimation of insufficient model}

\section{Experiment:}

- Left figure: estimation of model with $c_{-}$const and c_square (basic model)

- Right figure: estimation of model with only c_square (insufficient model)

- The results can prove (experimentally) the existence of a constant force!

- The figures show the best fits of the models.
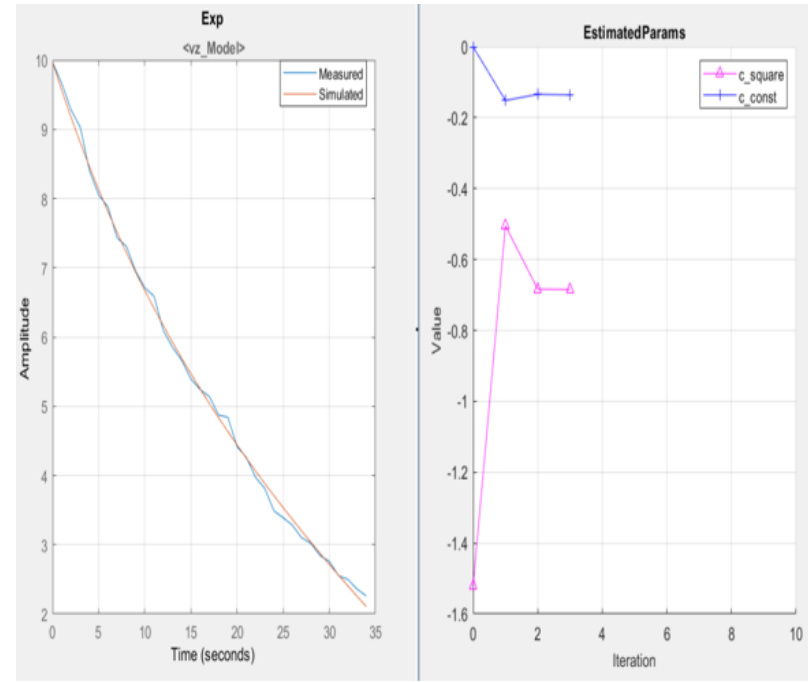

Estimation of 2 variables (c_const and c_square), $f=0.0035$
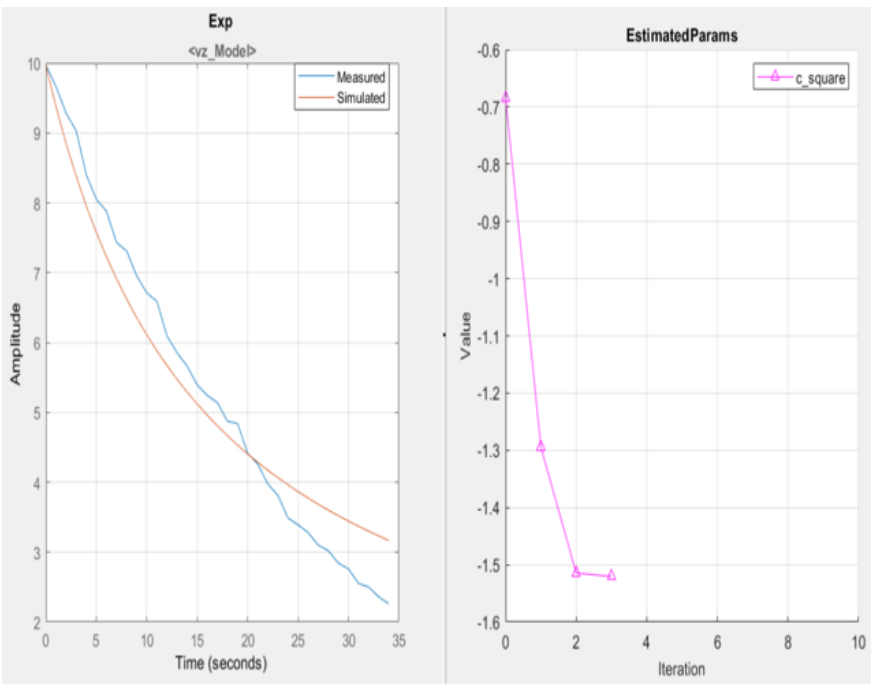

Estimation of 1 variable (c_square), $\mathrm{f}=0.097$ (28x larger)

Figure 5: Estimation of insufficient model experiment. The fit of the insufficient model (right) is mediocre and the value of its objective function is 28 times larger than the objective function value of the basic model (left). 
International Journal of Engineering and Management Sciences (IJEMS) Vol. 5. (2020). No. 2

DOI: 10.21791/IJEMS.2020.2.14.

\section{Conclusions}

\section{Conclusion of the method of parameter estimation:}

- Parameter estimation is a valid alternative when expensive measurement devices are not accessible.

- For example, a wind tunnel and a torque sensor were not accessible for this project.

\section{Conclusion of the application of parameter estimation for this project:}

- Advantages:

- Can be performed with affordable devices

- Vehicle is not required to be taken apart

- Absolute method: the estimated parameters contain all energy losses in a real environment (not just the drag and rolling resistance)

○ The performed measurements could be used for validating the parameters (after we measured the parameters with other methods)

- Disadvantages:

○ Relatively long and flat track is needed with good weather conditions

- Some kind of clutch is needed (the force losses of the engine and the driveline are meant to be modelled elsewhere)

- Accuracy is limited because of the method and the disturbances of the real environment

○ Forces are not measured directly, only estimated

\section{Acknowledgments}

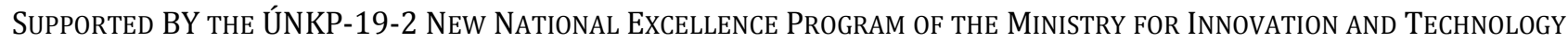

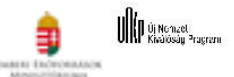

\section{References}

[1] Beck, J.V. and Arnold, K.J., 1977. Parameter estimation in engineering and science. James Beck.

[2] Yang, W.Y., Cao, W., Chung, T.S. and Morris, J., 2005. Applied numerical methods using MATLAB. John Wiley \& Sons.

[3] Jensen, J.C., Chang, D.H. and Lee, E.A., 2011, July. A model-based design methodology for cyberphysical systems. In 2011 7th International Wireless Communications and Mobile Computing Conference (pp. 1666-1671). IEEE. 
International Journal of Engineering and Management Sciences (IJEMS) Vol. 5. (2020). No. 2

DOI: 10.21791/IJEMS.2020.2.14.

[4] Bucaioni, A., Addazi, L., Cicchetti, A., Ciccozzi, F., Eramo, R., Mubeen, S. and Sjödin, M., 2018. MoVES: A Model-driven Methodology for Vehicular Embedded Systems. IEEE Access, 6, pp.64246445.

[5] https://en.wikipedia.org/wiki/Lumped-element_model (downloaded: 10/25/2019)

[6] https://en.wikipedia.org/wiki/Distributed_parameter_system (downloaded: 10/25/2019)

[7] Tritton, D.J., 2012. Physical fluid dynamics. Springer Science \& Business Media.

[8] Bae, H.S., Ryu, J. and Gerdes, J.C., 2001, August. Road grade and vehicle parameter estimation for longitudinal control using GPS. In Proceedings of the IEEE Conference on Intelligent Transportation Systems (pp. 25-29).

[9] Pacejka, Hans. Tire and vehicle dynamics. Elsevier, 2005.

[10] Szántó, A. and Hajdu, S. (2019), Vehicle Modelling and Simulation in Simulink. International Journal of Engineering and Management Sciences (IJEMS) Vol. 4. (2019). No. 1 DOI: 10.21791/IJEMS.2019.1.33. 